\title{
A variant of Runx2 that differs from the bone isoform in its splicing is expressed in spermatogenic cells
}

Satoru Kanto, Marcin Grynberg, Yoshiyuki Kaneko, Jun Fujita, Masanobu Satake

Background. Members of the Runx gene family encode transcription factors that bind to DNA in a sequence-specific manner. Among the three Runx proteins, Runx2 comprises 607 amino acid (aa) residues, is expressed in bone, and plays crucial roles in osteoblast differentiation and bone development. We examined whether the Runx2 gene is also expressed in testes.

Methods. Murine testes from 1-, 2-, 3-, 4-, and 10-week-old male mice of the C57BL/6] strain and $W / W^{v}$ strain were used throughout the study. Northern Blot Analyses were performed using extracts form the murine testes. Sequencing of cDNA clones and 5'-Rapid Amplification of cDNA Ends were performed to determine the full length of the transcripts, which revealed that the testicular Runx2 comprises 106 aa residues coding novel protein. Generating an antiserum using the amino-terminal 15 aa of Runx2 (Met ${ }^{1}$ to Gly ${ }^{15}$ ) as an antigen, immunoblot analyses were performed to detect the predicted polypeptide of 106 aa residues with the initiating Met $^{1}$. With the affinity-purified anti-Runx2 antibody, immunohistochemical analyses were performed to elucidate the localization of the protein. Furthermore, bioinformatic analyses were performed to predict the function of the protein. Results. A Runx2 transcript was detected in testes and was specifically expressed in germ cells. Determination of the transcript structure indicated that the testicular Runx2 is a splice isoform. The predicted testicular Runx2 polypeptide is composed of only 106 aa residues, lacks a Runt domain, and appears to be a basic protein with a predominantly alpha-helical conformation. Immunoblot analyses with an anti-Runx2 antibody revealed that Met $^{1}$ in the deduced open reading frame of Runx2 is used as the initiation codon to express an $11 \mathrm{kDa}$ protein. Furthermore, immunohistochemical analyses revealed that the Runx2 polypeptide was located in the nuclei, and was detected in spermatocytes at the stages of late pachytene, diplotene and second meiotic cells as well as in round spermatids. Bioinformatic analyses suggested that the testicular Runx 2 is a histone-like protein.

Discussion. A variant of Runx2 that differs from the bone isoform in its splicing is expressed in pachytene spermatocytes and round spermatids in testes, and encodes a histone-like, nuclear protein of 106 aa residues. Considering its nuclear localization and 
differentiation stage-dependent expression, Runx2 may function as a chromatinremodeling factor during spermatogenesis. We thus conclude that a single Runx2 gene can encode two different types of nuclear proteins, a previously defined transcription factor in bone and cartilage and a short testicular variant that lacks a Runt domain. 


\section{Title page}

2 A variant of Runx2 that differs from the bone isoform in its splicing is expressed in

3 spermatogenic cells

5 Satoru Kanto a, b, *, Marcin Grynberg ${ }^{\text {c, d }}$, Yoshiyuki Kaneko e , Jun Fujita e , Masanobu Satake ${ }^{\text {a }}$

7 a Department of Molecular Immunology, Institute of Development, Aging and Cancer, Tohoku

8 University, Sendai, Japan; ${ }^{b}$ Department of Urology, Graduate School of Medicine, Tohoku

9 University, Sendai, Japan; 'Program in Bioinformatics and Systems Biology, Stanford Burnham

10 Medical Research Institute, La Jolla, CA, USA; Institute of Biochemistry and Biophysics, Polish

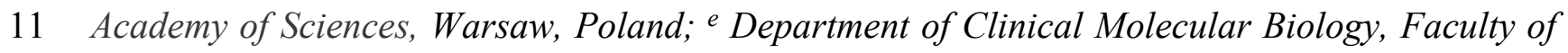

12 Medicine, Kyoto University, Kyoto, Japan

14 Abbreviations: aa, amino acid; GST, glutathione-S-transferase; ORF, open reading frame; PBS, 15 phosphate-buffered saline.

17 *Corresponding author at: Department of Urology, Yamagata Tokushukai Hospital, 2-3-51

18 Kiyosumimachi, Yamagata, 990-0834 Japan. Tel: +81-23-647-3434. Fax: +81-23-647-3400.

19 Email: s.kanto@yamatoku-hp.jp

20 Running title: Novel variant of Runx2 is expressed in testis 


\section{Abstract}

23 Background. Members of the Runx gene family encode transcription factors that bind to DNA in

24 a sequence-specific manner. Among the three Runx proteins, Runx2 comprises 607 amino acid

25 (aa) residues, is expressed in bone, and plays crucial roles in osteoblast differentiation and bone

26 development. We examined whether the Runx2 gene is also expressed in testes.

27 Methods. Murine testes from 1-, 2-, 3-, 4-, and 10-week-old male mice of the C57BL/6J strain and

$28 \mathrm{~W} / \mathrm{W}^{\mathrm{v}}$ strain were used throughout the study. Northern Blot Analyses were performed using

29 extracts form the murine testes. Sequencing of cDNA clones and 5'-Rapid Amplification of cDNA

30 Ends were performed to determine the full length of the transcripts, which revealed that the

31 testicular Runx2 comprises 106 aa residues coding novel protein. Generating an antiserum using

32 the amino-terminal 15 aa of Runx2 $\left(\operatorname{Met}^{1}\right.$ to Gly ${ }^{15}$ ) as an antigen, immunoblot analyses were

33 performed to detect the predicted polypeptide of 106 aa residues with the initiating $\mathrm{Met}^{1}$. With the

34 affinity-purified anti-Runx2 antibody, immunohistochemical analyses were performed to

35 elucidate the localization of the protein. Furthermore, bioinformatic analyses were performed to

36 predict the function of the protein.

37 Results. A Runx2 transcript was detected in testes and was specifically expressed in germ cells.

38 Determination of the transcript structure indicated that the testicular Runx2 is a splice isoform. The

39 predicted testicular Runx2 polypeptide is composed of only 106 aa residues, lacks a Runt domain,

40 and appears to be a basic protein with a predominantly alpha-helical conformation. Immunoblot 
41 analyses with an anti-Runx2 antibody revealed that $\mathrm{Met}^{1}$ in the deduced open reading frame of

42 Runx2 is used as the initiation codon to express an $11 \mathrm{kDa}$ protein. Furthermore,

43 immunohistochemical analyses revealed that the Runx2 polypeptide was located in the nuclei, and

44 was detected in spermatocytes at the stages of late pachytene, diplotene and second meiotic cells as

45 well as in round spermatids. Bioinformatic analyses suggested that the testicular Runx 2 is a

46 histone-like protein.

47 Discussion. A variant of Runx2 that differs from the bone isoform in its splicing is expressed in

48 pachytene spermatocytes and round spermatids in testes, and encodes a histone-like, nuclear

49 protein of 106 aa residues. Considering its nuclear localization and differentiation stage-dependent

50 expression, Runx 2 may function as a chromatin-remodeling factor during spermatogenesis. We

51 thus conclude that a single Runx2 gene can encode two different types of nuclear proteins, a

52 previously defined transcription factor in bone and cartilage and a short testicular variant that lacks

53 a Runt domain.

Introduction

56 Runx transcription factors are characterized by the presence of a Runt domain (Kagoshima et al.,

57 1993). This domain contains 130 amino acids (aa) and is responsible for sequence-specific DNA-

58 binding activity and for dimerization with the protein PEBP2 $\beta / C B F \beta$. There are three known Runx

59 genes in mammals, Runx1, Runx2, and Runx3, each of which plays important roles in cell

60 proliferation and differentiation as well as in the occurrence of specific human diseases (Wong et 
61 al., 2011; Chuang, Ito \& Ito, 2013).

62 Runx2, the topic of this study, is expressed in bone, thymus, testis, and brain (Satake et al.,

63 1995; Komori et al., 1997; Otto et al., 1997; Jenog et al., 2008). In bone and thymus, the Runx2

64 transcript contains a Runt domain sequence and the translated product functions as a transcription

65 factor. In bone, gene-targeting studies have demonstrated that Runx2 is essential for the

66 differentiation of immature osteoblasts into mature osteocytes. In mice lacking the Runt domain of

67 Runx2, ossification of the skeletal system is severely impaired and the animals die soon after birth

68 due to a respiratory defect (Komori et al., 1997). Of clinical importance, heterozygous loss of

69 Runx2 causes cleidocranial dysplasia in humans, which is characterized by hypoplasia/aplasia of

70 the clavicles and fontanelles (Otto et al., 1997; Mundlos et al., 1997).

In the thymus, Runx2 appears to function as an oncogene because the insertion of a

retroviral genome near to the Runx2 locus in mice results in its overexpression and subsequently

73 the occurrence of T-cell leukemia (Stewart et al., 1997). In addition, overexpression of a Runx2

74 transgene in the T-cell lineage perturbs the differentiation of thymocytes, mainly at the $\beta$ selection

75 stage, and produces a population that predominantly consists of immature CD8+ thymocytes

76 (Vaillant et al., 2002).

Runx2 is also expressed in the testis. This was originally reported by Satake et al. (Satake et

al., 1995) and subsequently by Ogawa et al. (Ogawa et al., 2000). According to Ogawa et al.

79 (Ogawa et al., 2000), the testicular Runx2 transcript displays several unique features. First, it is

80 remarkably shorter $(\sim 1.8 \mathrm{~kb})$ than the transcripts found in bone (6.3 and $7.4 \mathrm{~kb})$, mainly due to the 
81 premature termination of the testicular transcript within exon 8. Second, as a result of alternative

82 splicing and fusion between exons 1 and 3, a new stop codon is generated in exon 3 . The deduced

83 open reading frame (ORF) encodes a polypeptide of only 106 aa residues. In addition, there are

84 two methionine codons within exon 1 of this ORF, Met ${ }^{1}$ and Met $^{69}$. Ogawa et al. (Ogawa et al.,

85 2000) predicted that $\mathrm{Met}^{69}$ is the translation initiation codon because the nucleotide sequence

86 adjacent to Met $^{69}$ is in better agreement with Kozak's rule than the sequence adjacent to Met $^{1}$

87 (Kozak, 2002). However, if Met $^{69}$ was the start codon, then the encoded polypeptide would only

88 be 38 aa residues long. Furthermore, because the alternative splicing removes exon 2, which

89 encodes the amino-terminal portion of the Runt domain, the testicular Runx 2 transcript cannot

90 encode a Runt domain-containing transcription factor.

In this study, we investigated the possibility that $\mathrm{Met}^{1}$ rather than $\mathrm{Met}^{69}$ is used as the

92 initiation codon for the translation of the testicular Runx2 transcript because the environment for

93 translation in testicular cells is distinct from that in somatic cells. Furthermore, we examined the

94 expression pattern of the putative 106-aa polypeptide in relation to the differentiation stages of

95 testicular germ cells. We propose that the single Runx2 gene can encode two distinct types of

96 protein: a small protein expressed in the testis that lacks a Runt domain, and a previously defined

97 Runt-containing transcription factor that is expressed in bone and thymus.

99 Materials and methods

100 Mice were maintained in the Animal Facility of the Institute of Development, Aging, and Cancer, 
101 Tohoku University, an environmentally controlled and specific pathogen-free facility. Animal

102 protocols were reviewed and approved by the Animal Studies Committee of the Tohoku

103 University (relevant approval number: 2013-IDAC-Animal-013).

104

\section{Northern Blot Analysis}

106 Testes were isolated from 1-, 2-, 3-, 4-, and 10-week-old male mice of the C57BL/6J strain and

107 from 10-week-old male mice of the $\mathrm{W} / \mathrm{W}^{\mathrm{v}}$ strain. Spermatocyte and spermatid fractions were

108 prepared from the cell suspension of C57BL/6J testes (Mays-Hoopes et al., 1995). Total

109 cytoplasmic RNA was prepared from testes using Isogen (Nippon Gene, Toyama, Japan).

110 Poly(A) ${ }^{+}$RNAs were selected using Oligo(dT)-Latex (Takara, Otsu, Japan) and $2 \mu g$ of sample

111 was electrophoresed through a $1 \%(\mathrm{w} / \mathrm{v})$ agarose gel containing $2.2 \mathrm{M}$ formaldehyde. RNA was

112 transferred from the gel to a membrane, and the membrane was hybridized with a ${ }^{32} \mathrm{P}$-labeled

113 probe as described previously (Chiba et al., 1997). The probes were prepared either from a

114 HindIII-NotI fragment of murine Runx2 cDNA (corresponding to nt 282 through to nt 473 in

115 NM_001146038.2) or murine Runx1 cDNA sequence, murine PEBP2 $\beta / C B F \beta$ cDNA sequence

116 and $\beta$-actin (Satake et al., 1995).

118 cDNA Cloning and Sequencing

119 A cDNA library prepared from murine testicular poly $(\mathrm{A})^{+}$RNA was provided by Y $^{\text {Nishina }}$

120 (Osaka University, Osaka, Japan). A ${ }^{32}$ P-labeled HindIII-NotI fragment of murine Runx2 cDNA 
121 was used as the probe. The library was screened under a stringent condition according to the

122 standard method. The cDNA inserts from each of the five isolated clones were excised from the

123 pAP3neo vector and subcloned into the pBluescript II vector. The entire length of the insert was

124 sequenced using the dideoxy-dye terminator method.

125

126 5'-Rapid Amplification of cDNA Ends (5'-RACE)

127 To determine the full length of the transcripts, 5'-RACE was performed following the manual

128 supplied by the manufacturer (Life Technologies). A gene-specific primer (5'-

129 TGTAAATACTGCTTGCAGCC-3') was annealed to poly(A) ${ }^{+}$RNAs and cDNA was synthesized.

130 After degrading RNA with RNase H, purified cDNA was tailed with dCTP and TdT. The dC-

131 tailed cDNA was amplified with the anchor primer and a nested gene-specific primer (5'-

132 GTGACCTGCAGAGATTAACC-3'). The double-stranded cDNA was subcloned into the

133 pBluescript II vector and sequenced.

\section{Bioinformatic Analysis}

136 The subcellular localizations of proteins were predicted using the PSORT program (Nakai \&

137 Horton, 1999). The secondary structures of proteins were predicted using the PSIPRED

138 (McGuffin, Bryson \& Jones, 2000), SAM-T99-2d (Karplus et al., 1999), and Profsec (Rost \&

139 Eyrich, 2001) programs. These programs were downloaded from the BioInfo MetaServer

140 (http://bioinfo.pl/). The PSI-BLAST program (Altschul et al., 1997) was used for homology 
141 searches. Three iterations were used before full saturation was reached. The domain architecture of

142 proteins was analyzed using the SMART tool (Letunic et al., 2002).

143

144 Immunoblot Analysis

145 Proteins were extracted from testes using RIPA buffer and $10 \mu \mathrm{g}$ of the sample was subjected to

$1468 \%$ or $10 \%(\mathrm{w} / \mathrm{v})$ SDS-polyacrylamide gel electrophoresis (PAGE). The ORF of the testicular

147 Runx2, which encodes a polypeptide of 106 aa, was fused in frame to glutathione-S-transferase

148 (GST) using the pGEX vector. The plasmid was transfected into E. coli and transformed bacteria

149 were lysed in sample buffer after induction with isopropyl- $\beta$-D-thiogalactoside. Two micrograms

150 of protein was separated by SDS-PAGE. Proteins were transferred from the gel to a membrane and

151 the blotted membranes were blocked with TBS-T buffer, which contained $20 \mathrm{mM}$ Tris-HCl $\mathrm{pH} 7.4$,

$152150 \mathrm{mM} \mathrm{NaCl}$, and $0.1 \%(\mathrm{v} / \mathrm{v})$ Tween 20 . The primary antibody was anti-Runx2 serum, which was

153 raised in rabbit using the amino-terminal 15 aa residues (MLHSPHKQPQNHKCG) of the murine

154 testicular Runx2 as an antigen. In some cases, the antiserum was preabsorbed with an excess

155 amount of antigen peptide. The secondary antibody was alkaline phosphatase-conjugated goat

156 anti-rabbit IgG (Promega, Madison, WI). The antibodies were diluted appropriately in TBS-T.

157 Immunologically reacted products were detected using the BCIP/NBT Color Development

158 Substrate Kit (Promega, Madison, WI).

159

160 Immunohistochemistry of Testicular Preparations 
161 To prepare frozen sections, testes from adult male C57BL/6J mice were cut into three pieces and

162 fixed in Zamboni solution for $6 \mathrm{hr}$ at $4^{\circ} \mathrm{C}$ with agitation. The tissues were immersed sequentially in

$16310 \%(\mathrm{w} / \mathrm{v}), 15 \%$, and $20 \%$ sucrose solutions prepared in phosphate-buffered saline (PBS) for $2 \mathrm{hr}$

164 each at $4^{\circ} \mathrm{C}$; embedded in OCT compound (Miles Laboratories, Berkeley, CA); and kept frozen at

$165-80^{\circ} \mathrm{C}$ until use. The tissues were cryostat-sectioned (7- $\mu \mathrm{m}$ thick sections), air-dried, and post-

166 fixed for $20 \mathrm{~min}$ in $4 \%(\mathrm{w} / \mathrm{v})$ paraformaldehyde prepared in PBS.

167 The sections were then treated with methanol containing $0.3 \%(\mathrm{v} / \mathrm{v})$ hydrogen peroxide

168 for $15 \mathrm{~min}$ followed by PBS containing 3\% (w/v) skimmed milk and 10\% (v/v) goat serum for 30

169 min. In immunohistochemistry, the anti-serum was affinity-purified using a peptide

170 (MLHSPHKQPQNHKCG)-linked Sepharose 4B column. The sections were incubated with

171 appropriately diluted, affinity-purified anti-Runx 2 antibody at $4{ }^{\circ} \mathrm{C}$ overnight, followed by

172 biotinylated goat anti-rabbit IgG for $30 \mathrm{~min}$. The primary antibody was detected using an ABC Kit

173 (Vector Laboratories, Burlingame, CA). The sections were post-stained with methyl green, and

174 coverslips were mounted on glass slides.

175

176 Results

177 The Testicular Runx2 Is Transcribed Specifically in Germ Cells

178 To gain insight into the significance of the Runx 2 transcript in testes, we first used $\mathrm{W} / \mathrm{W}^{\mathrm{v}}$ mice and

179 wild-type C57BL/6 mice. W/Wv mice lack germ cells except spermatogonia because of mutations

180 in the $c$-Kit gene (Kubota et al., 2009). RNA was extracted from testes and processed for Northern 
181 blot analysis (Fig. 1, Fig. S1). The Runx2 transcript was detected as a broad band of $\sim 1.8 \mathrm{~kb}$ length

182 in wild-type testis, but not in $\mathrm{W} / \mathrm{W}^{\mathrm{v}}$ testis (Fig. 1, A, lanes 1 and 2). By contrast, Runxl transcript

183 (Fig. S1), $P E B P 2 \beta / C B F \beta$ transcript (Fig. 1, $B$, lanes 1 and 2) and $\beta$-actin transcript (Fig. S1) used

184 as loading control, were detected in both RNA samples tested. This indicates that the testes-

185 specific Runx2 transcript is expressed in germ cells, not in somatic cells in testes.

186 Next, spermatocytes at the pachytene stage and spermatids were purified from a cell

187 suspension prepared from wild-type testis, and then RNA analyzed. The Runx2 transcript was

188 detected in both the spermatocyte and spermatid fractions (Fig. 1, A, lanes 3 and 4). The

189 differentiation of germ cells proceeds in a synchronous fashion immediately after birth; therefore,

190 RNA was prepared from the testes of newborn mice and analyzed (Fig. 1, A, lanes 5-8). A band of

$1911.8 \mathrm{~kb}$ was detected in 4 week-old testis, which largely contains germ cells at the spermatid stage.

192 Thus, the testicular Runx2 was transcribed in germ cells at the spermatocyte and spermatid stages.

\section{Determination of The Structure of The Testicular Runx2 Transcript}

195 Although the study by Ogawa et al. predicted the ORF of Runx2 to encode a 106-aa protein

196 (Ogawa et al., 2000), the cDNA and aa sequences have not been published or registered in a public

197 database. Only the junction sequence between exons 1 and 3 is available in the literature.

198 Therefore, we decided to independently clone Runx2 cDNAs from a library prepared from murine

199 testis. The nucleotide sequence that was determined from the obtained cDNA clones and 5'RACE

200 is presented in Figure 2, $A$ and has been deposited at NCBI (accession number, DQ458792). The 
201 AUG codons of $\mathrm{Met}^{1}$ and $\mathrm{Met}^{69}$ as well as the termination codon are indicated in bold. that are separated by an intronic sequence of 197 nucleotides. We tentatively designated these each exon is shown in Figure 2, $B$. Exon $1 \mathrm{U}$ harbors the $\mathrm{Met}^{1}$ codon, whereas exon 1D includes the Met $^{69}$ codon. Exons $1 \mathrm{U}$ and 1D are also transcribed in bone Runx2 (Xiao et al., 1998) and the ORF of bone Runx2 has been predicted (NM_009820) (Ducy et al., 1997). The amino-terminal 87 aa residues are common to both the testicular and bone ORFs, whereas the carboxy-terminal 19 aa residues derived from exon 3 are unique to the testicular ORF.

\section{Bioinformatic Analysis Suggests that The Testicular Runx2 Variant Is Authentic}

213 The predicted aa sequence of the testicular Runx2 variant is shown in Figure $3, A$. We performed

214 bioinformatic analysis as a first step to examine the theoretical likelihood that this is an authentic

215 protein. According to the PSORTII program, the Runx2 variant had a 43\% probability of being a

216 nuclear protein. Moreover, the variant appears to be a basic protein; out of 106 aa residues, 22 are

217 basic and only 6 are acidic. Secondary structure assignment programs suggested that Runx 2 has

218 both $\alpha$-helical and $\beta$-sheet structures (indicated by $\mathrm{H}$ and $\mathrm{E}$, respectively; Fig. 3, $A$ ). In particular, a

219 stretch of 55 aa in the amino-terminus are folded into two distinct $\alpha$-helices. A basic, nuclear

220 protein with a high $\alpha$-helical content is reminiscent of a histone. On the other hand, three carboxy- 
221 terminal Cys residues may adopt a globular structure with a sulfur bridge (indicated by green, Fig.

$2223, A)$.

223 The amino-terminal part of the Runx2 variant (from $\mathrm{Met}^{1}$ to $\mathrm{Ser}^{46}$ ) showed weak but

224 significant similarity to a 48-aa sequence of a murine protein of unknown function that is

225 expressed in the eye, namely, Q7TPL8/33942100 (SWISS and NCBI IDs, respectively; Fig. 3, B).

226 Q7TPL8 possesses features that suggest it is a transcription factor (Fig. 3, C). At the amino-

227 terminus, it contains a KRAB (Kruppel-associated box) domain that may function as a

228 transcription-repression domain, and at the carboxy-terminus, it harbors eight zinc fingers that

229 may function as nucleic acid-binding structures. The region of Q7TPL8 that shows similarity to

230 Runx2 is located immediately before the stretch of zinc fingers. This feature suggests that the

231 amino-terminal motif of 46 aa residues in Runx2 represents a functional domain. In addition, the

232 testicular Runx2 also retains a stretch of 19 aa residues (from $\mathrm{Met}^{69}$ to $\operatorname{Trp}^{87}$ ) that corresponds to an

233 important transcription activation domain in bone Runx2 (Thirunavukkarasu et al., 1998).

234 Taken together, these observations increase the probability that the testicular Runx2 is an

235 authentic protein.

\section{Met $^{\mathbf{1}}$ Is Used as An Initiation Codon in The Testicular Runx2 Variant}

238 We next examined whether Met $^{1}$ is indeed used as an initiation codon in testis. We generated an

239 antiserum using the amino-terminal 15 aa of Runx2 (Met ${ }^{1}$ to Gly $\left.{ }^{15}\right)$ as an antigen. A protein extract

240 was prepared from mouse testis and processed for immunoblot analysis. A clear band of 
241 approximately $11 \mathrm{kDa}$ was detected (Fig. 4, $A$, lane 1 , indicated by an arrow), whereas inclusion of

242 an excess amount of antigen peptide in the immunoreaction abolished this band (Fig. 4, A, lane 2).

243 Lysates from bacteria expressing a GST-Runx2 ORF fusion protein were run as controls in lanes 3

244 and 4, and GST-Runx2 was detected with the same antiserum. It is therefore highly likely that this

245 ORF is expressed in testis as a polypeptide of 106 aa residues.

246

247 The Testicular Runx2 Is A Differentiation Stage-dependent Nuclear Protein

248 To verify that the truncated variant of Runx2 is expressed, we performed immunohistochemistry

249 on frozen sections of testes (Fig. 4, B). Panel a shows the staining pattern in mouse testis that was

250 probed with the affinity-purified anti-Runx2 antibody raised against the Met $^{1}$-to-Gly ${ }^{15}$ peptide.

251 Positively stained cells were detected within seminiferous tubules. This immunostaining was

252 specific for Runx2 because it completely disappeared when the antibody was preabsorbed with an

253 excess amount of antigen peptide (panel b).

254 Interestingly, the distribution pattern of positive cells appeared to differ from tubule to

255 tubule. Therefore, each seminiferous tubule containing positive cells were classified according to

256 the differentiation stage. This was judged by the morphology of cells and nuclei (see panel c-i).

257 Runx2 staining was detected in the following differentiation stages: late pachytene spermatocytes

258 at stages VIII and X (panels c and d), diplotene spermatocytes at stage XI (panel e), cells of second

259 meiotic phase at stage XII (panel f), and round spermatids of spermiogenesis phase 1, 2/3, and 5 at

260 stage I, II/III, and V, respectively (panels g, h, and i). In short, the Runx2 variant was detected in 
261 various stages of differentiation, from late pachytene spermatocytes to round spermatids. It must

262 be noted that detection of Runx 2 protein by immunohistochemistry (Fig. $4, B$ ) and detection of the

263 Runx2 transcript by Northern blot (Fig. 1) coincides in terms of germ cell specific expression and

264 expression in pachytene spermatocytes to spermatids.

265 Finally, as seen in the enlarged view in Figure 4, $C$, positive staining was always restricted

266 to the nuclei, indicating that the testicular Runx2 is a nuclear protein. The protein was detected as

267 multiple foci in the nuclei of germ cells. The original picture of immunohistochemistry observed

268 under differential interference contrast microscope can be seen as supplement figures (Fig. S2, Fig.

269 S3).

270

271 Discussion

272 This study showed that the testicular Runx2 is located in the nucleus, appears to be a basic protein,

273 and has a predominantly $\alpha$-helical conformation. These characteristics are somewhat reminiscent

274 of histone proteins. The protein was detected in spermatocytes at the late pachytene and diplotene

275 stages as well as in round spermatids. At the pachytene and diplotene stages, genetic information is

276 exchanged between a pair of homologous chromosomes through homologous recombination.

277 Thereafter, in post-meiotic and round spermatids, chromatins containing a haploid genome start

278 preparing to remodel their structures. The testicular Runx2 protein detected in this study might be

279 involved in the aforementioned processes. In this context, it is worth noting the histone variants

280 that are expressed specifically in male germ cells. For example, a testicular variant of linker 
281 histone 1 is detected in pachytene spermatocytes and persists until the round spermatid stage

282 (Brock, Trostle \& Meistrich, 1980; Drabent et al., 1996). Likewise, TH2A and TH2B (testicular

283 variants of the core histones $\mathrm{H} 2 \mathrm{~A}$ and $\mathrm{H} 2 \mathrm{~B}$ ) and $\mathrm{H} 3 \mathrm{t}$ (a testicular variant of the core histone $\mathrm{H} 3$ )

284 are expressed in round spermatids. It would be interesting to determine whether the testicular

285 Runx2 is incorporated into nucleosomes and play roles in loosening their structures, as histone

286 variants are suggested to do (Rathke et al., 2014). In any case, a single Runx2 gene can encode two

287 different types of nuclear proteins, a previously defined transcription factor in bone and cartilage

288 and a short testicular variant that lacks a Runt domain. The amino-terminal 87 aa residues shared

289 by the testicular and bone Runx 2 might exert distinct functions. Namely, the former might

290 represent a histone-like protein whereas the latter likely represents a transcription activation

291 domain.

292

Jeong et al. reported the expression of the Runx2 transcription factor in mice sperm (Jeong

293 et al., 2008). Their immunoblot analyses using a monoclonal antibody against Runx2 detected

294 proteins of 47 and $65 \mathrm{kD}$ in lysates from testes and sperm. Therefore, the observations of Jeong et

295 al. (Jeong et al., 2008) appear to be substantially different from our results. However, it must be

296 noted that our Northern blot analyses could detect, albeit faintly, bands larger than the major 1.8-

$297 \mathrm{~kb}$ band (see fig. 1). If such larger transcripts harbor the Runt domain sequence, they probably

298 encode the Runx transcription factor that is found in bone and T cells. Thus, the findings of Jeong

299 et al. (Jeong et al., 2008) and the current study may not be contradictory.

300 Although this study used murine testes, the Runx2 gene is conserved among mammals, 
301 thus suggesting a possible extrapolation of our findings to other species. In this sense, we note that

302 the two homologous transcripts are found in the NCBI database. They are AB573882.1 and

303 AB573881.1, and are reported to be expressed in a periosteum tissue of rat. In these rat transcripts,

304 splicing appears to skip exon 2 and fuse exons 1 and 3 as in the same way as a murine testicular

305 Runx2 transcript. Whether a protein is expressed from this rat Runx2 transcript is not known at

306 present.

307

308 Conclusion

309 In conclusion, a variant of Runx2 that differs from the bone isoform in its splicing is

310 expressed in pachytene spermatocytes and round spermatids in murine testes, and encodes a

311 histone-like, nuclear protein of 106 amino acid residues. Considering its nuclear localization and

312 differentiation stage-dependent expression, Runx 2 may function as a chromatin-remodeling factor

313 during spermatogenesis.

314

315 Acknowledgments: We are grateful to Dr. Y. Nishina for providing a cDNA library from murine

316 testis and to Dr. H. Kawashima for valuable advice on antibody affinity-purification.

318 References

319 Altschul SF, Madden TL, Schäffer AA, Zhang J, Zhang Z, Miller W, Lipman DJ. 1997.

320 Gapped BLAST and PSI-BLAST: a new generation of protein database search programs. Nucl 
321 Acids Res 25: 3389-3402.

322 Brock WA, Trostle PK, Meistrich ML. 1980. Meiotic synthesis of testis histones in the rat. Proc

323 Natl Acad Sci USA 77: 371-375.

324 Chiba N, Watanabe T, Nomura S, Tanaka Y, Minowa M, Niki M, Kanamaru R, Satake M.

325 1997. Differentiation dependent expression and distinct subcellular localization of the

326 protooncogene product, $\mathrm{PEBP} 2 \beta / \mathrm{CBF} \beta$, in muscle development. Oncogene 14: 2543-2552.

327 Chuang LS, Ito K, Ito Y. 2013. RUNX family: Regulation and diversification of roles through

328 interacting proteins. International Journal of Cancer 132: 1260-1271. DOI: 10.1002/ijc.27964.

329 Drabent B, Bode C, Bramlage B, Doenecke D. 1996. Expression of the mouse testicular histone

330 gene H1t during spermatogenesis. Histochem Cell Biol 106: 247-251.

331 Ducy P, Zhang R, Geoffroy V, Ridall AL, Karsenty G. 1997. Osf2/Cbfa1: a transcriptional

332 activator of osteoblast differentiation. Cell 89: 747-754.

333 Jeong JH, Jin JS, Kim HN, Kang SM, Liu JC, Lengner CJ, Otto F, Mundlos S, Stein JL, van

334 Wijnen AJ, Lian JB, Stein GS, Choi JY. 2008. Expression of Runx2 transcription factor in non-

335 skeletal tissues, sperm and brain. J Cell Physiol 217: 511-517. DOI: 10.1002/jcp.21524.

336 Kagoshima H, Shigesada K, Satake M, Ito Y, Miyoshi H, Ohki M, Pepling M, Gergen P. 1993.

337 The Runt domain identifies a new family of heteromeric transcriptional regulators. Trends in

338 Genetics 9: 338-341.

339 Karplus K, Barrett C, Cline M, Diekhans M, Grate L Hughey R. 1999. Predicting protein

340 structure using only sequence information. Proteins Suppl 3 :121-125. 
341 Komori T, Yagi H, Nomura S, Yamaguchi A, Sasaki K, Deguchi K, Shimizu Y, Bronson RT,

342 Gao YH, Inada M, Sato M, Okamoto R, Kitamura Y, Yoshiki S, Kishimoto T. 1997. Targeted

343 disruption of $C b f a l$ results in a complete lack of bone formation owing to maturational arrest of

344 osteoblasts. Cell 89: $755-764$.

345 Kozak M. 2002. Pushing the limits of the scanning mechanism for initiation of translation. Gene

346 299: 1-34.

347 Kubota H, Avarbock MR, Schmidt JA, Brinster RL. 2009. Spermatogonial stem cells derived

348 from infertile $\mathrm{W}^{\mathrm{v}} / \mathrm{W}^{\mathrm{v}}$ mice self-renew in vitro and generate progeny following transplantation.

349 Biol Reprod 81: 293-301. DOI: 10.1095/biolreprod.109.075960.

350 Letunic I, Goodstadt L, Dickens NJ, Doerks T, Schultz J, Mott R, Ciccarelli F, Copley RR,

351 Ponting CP, Bork P. 2002. Recent improvements to the SMART domain-based sequence

352 annotation resource. Nucl. Acids Res 30: 242-244.

353 Mays-Hoopes LL, Bolen J, Riggs AD, Singer-Sam J. 1995. Preparation of spermatogonia,

354 spermatocytes, and round spermatids for analysis of gene expression using fluorescence-activated

355 cell sorting. Biol Repro 53 :1003-1011.

356 McGuffin LJ, Bryson K, Jones DT. 2000. The PSIPRED protein structure prediction server.

357 Bioinformatics 16: 404-405.

358 Mundlos S, Otto F, Mundlos C, Mulliken JB, Aylsworth AS, Albright S, Lindhout D, Cole

359 WG, Henn W, Knoll JH, Owen MJ, Mertelsmann R, Zabel BU, Olsen BR. 1997. Mutations

360 involving the transcription factor CBFA1 cause cleidocranial dysplasia. Cell 89: 773-779. 
361 Nakai K, Horton P. 1999. PSORT: a program for detecting sorting signals in proteins and

362 predicting their subcellular localization. Trends Biochem Sci 24: 34-35.

363 Ogawa S, Harada H, Fujiwara M, Tagashira S, Katsumata T, Takada H. 2000. Cbfal, an

364 essential transcription factor for bone formation, is expressed in testis from the same promoter

365 used in bone. DNA Res. 7: 181-185.

366 Otto F, Thornell AP, Crompton T, Denzel A, Gilmour KC, Rosewell IR, Stamp GW,

367 Beddington RS, Mundlos S, Olsen BR, Selby PB, Owen MJ. 1997. Cbfal, a candidate gene for

368 cleidocranial dysplasia syndrome, is essential for osteoblast differentiation and bone development.

369 Cell 89: 765-771.

370 Rathke C, Baarends WM, Awe S, Renkawitz-Pohl R. 2014. Chromatin dynamics during

371 spermiogenesis. Biochim Biophys Acta - Gene Reg Mech 1839: 155-168. DOI:

372 10.1016/j.bbagrm.2013.08.004.

373 Rost B, Eyrich VA. 2001. EVA: large-scale analysis of secondary structure prediction. Proteins

374 Suppl. 5: 192-199.

375 Satake M, Nomura S, Yamaguchi-Iwai Y, Takahama Y, Hashimoto Y, Niki M, Kitamura Y,

376 Ito Y. 1995. Expression of the Runt domain-encoding PEBP2 $\beta$ genes in $T$ cells during thymic

377 development. Mol Cell Biol 15: 1662-1670.

378 Stewart M, Terry A, Hu M, O'Hara M, Blyth K, Baxter E, Cameron E, Onions DE, Neil JC.

379 1997. Proviral insertions induce the expression of bone-specific isoforms of PEBP2 $\alpha A$ (CBFA1):

380 evidence for a new myc collaborating oncogene. Proc Natl Acad Sci USA 94: 8646-8651. 
381 Thirunavukkarasu K, Mahajan M, McLarren KW, Stifani S, Karsenty G. 1998. Two

382 domains unique to osteoblast-specific transcription factor Osf2/Cbfa1 contribute to its 383 transactivation function and its inability to heterodimerize with Cbf $\beta$. Mol Cell Biol 18: 4197-

3844208.

385 Vaillant F, Blyth K, Andrew L, Neil JC, Cameron ER. 2002. Enforced expression of Runx2

386 perturbs T cell development at a stage coincident with $\beta$-selection. J Immunol 169: 2866-2874.

387 Wong WF, Kohu K, Chiba T, Sato T, Satake M. 2011. Interplay of transcription factors in T-

388 cell differentiation and function: the role of Runx. Immunology 132: 157-164. DOI:

389 10.1111/j.1365-2567.2010.03381.x.

390 Xiao ZS, Thomas R, Hinson TK, Quarles LD. 1998. Genomic structure and isoform expression

391 of the mouse, rat and human Cbfal/Osf2 transcription factor. Gene 214: 187-197. 


\section{Figure 1 (on next page)}

Northern blot analysis of Runx2 expression in testis

Fig. 1- RNA was prepared from 10-week-old W/Wv and C57BL/6) mouse testes (lanes 1 and 2, respectively), from pachytene spermatocytes and round spermatids (lanes 3 and 4 , respectively), and from 1-, 2- 3- and 4-week-old C57BL/6J mouse testes (lanes 5, 6, 7 and 8, respectively). The probes were cDNAs of the murine Runx2 (A) (a HindIII-Notl fragment corresponding to nt 282 through to nt 473 in NM_001146038.2) and PEBP2 $\beta / C B F \beta$ (B). The numbers alongside the gels show the sizes of the transcripts in kb. 


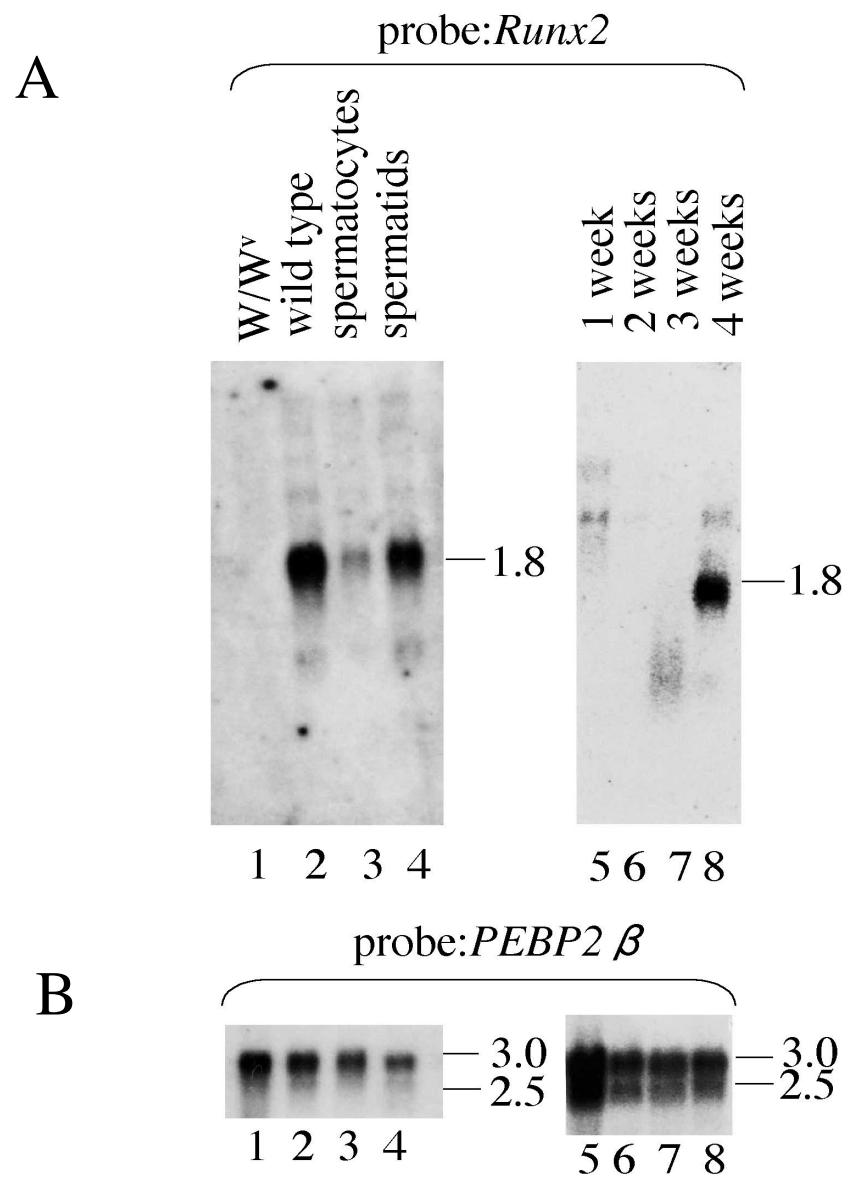


Figure 2 (on next page)

The nucleotide sequence and the structure of testicular Runx2

Fig. 2 - (A) The nucleotide sequence of the testicular Runx 2 transcript (1,480 nucleotides) is shown. The two ATG codons represent the Met1 and Met69. The TGA represents the termination codon. The two forward slashes indicate the boundaries between exons $1 \mathrm{U}$ and 1D and between exons 1D and 3. The underlined sequence is a poly(A)-addition signal. (B) Comparison of open reading frames that are assigned to the testis- and bone-derived Runx2 transcripts is shown. The numbers represent the exon numbers and the boxes represent the coding regions. 
A

aaaagattgagaaagagggagggaagagagcaagggggaagccacagtggtaggcagtcccactttactttgagt actgtgaggtcacaaaccacatcgattctgtctctccagtaatagtgcttgcaaaaataggagttttaaagctt ttgcttttttggattgtgtgaatgcttcattcgcctcacaaacaaccacagaaccacaagtgcggtgcaaacttt ctccaggaagactgcaagaaggctctggcgtttaaatggttaatctctgcaggtcactaccagccaccgagacca accgagtca/

tttaaggctgcaagcagtatttacaacagagggcacaagttctatctggaaaaaaaggagggactatggcgtca aacagcctcttcagcgcagtgacaccgtgtcagcaaagcttcttttgg/

ggttgtagccctcggagaggtaccagatgggactgtggttaccgtcatggccgggaatgatgagaactactccgc cgagctccgaaatgcctccgctgttatgaaaaccaagtagccaggttcaacgatctgagatttgtgggccggag cggacgaggcaagagtttcaccttgaccataacagtcttcacaaatcctccccaagtggccacttaccacagagc tattaaagtgacagtggacggtccccgggaaccaagaaggcacagacagaagcttgatgactctaaacctagttt gttctctgatcgcctcagtgatttagggcgcattcctcatcccagtatgagagtaggtgtcccgcctcagaaccc acggccctccctgaactctgcaccaagtccttttaatccacaaggacagagtcagattacagatcccaggcaggc acagtcttccccaccgtggtcctatgaccagtcttacccctcctatctgagccagatgacatccccatccatcca ctccaccacgccgctgtcttccacacggggcaccgggctacctgccatcactgacgtgcccaggcgtatttcaga tgatgacactgccacctctgacttctgcctctggccttcctctctcagtaagaagagccaggcaggtgcttcaga actgggcccttttcagaccccaggcagttcccaagcatttcatccctcactgagagccgcttctccaacccacg aatgcactacccagccacctttacctacaccccgccagtcacgtcaggcatgtccctcggcatgtccgccaccac tcactaccacacgtacctgccaccaccctaccccggctcttcccaaagccagagtggacccttccagaccagcag cactccatatctctactatggtacttcgtcagcatcctatcagttcccaatggtacccgggggagaccggtctcc ttccaggatggtcccaccatgcaccaccacctcgaatggcagcacgctattaaatccaaatttgcctaaccagaa aaaaaaaaaaaaaa

B

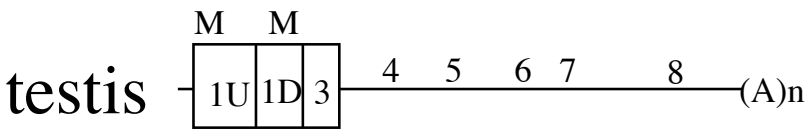

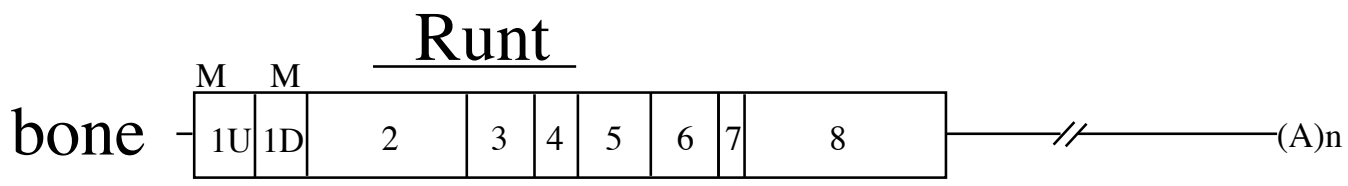


Figure 3 (on next page)

Bioinformatic analysis of the testicular Runx2 polypeptide

Fig. 3 - (A) Secondary structures such as $\alpha$-helices and $\beta$-sheets are indicated by $H$ and $E$, respectively. The two Met and five Cys residues are indicated. The two forward slashes indicate the boundaries of the exons. (B) A homologous motif found in testicular Runx2 and Q7TPL8 proteins is shown. Identical or similar aa are indicated. (C) The domain architecture of the Q7TPL8 protein is shown. The circle and boxes represent the KRAB and C2H2-type zinc finger domains, respectively. The region showing similarity to testicular Run 2 is indicated by a red line. 


\section{A testicular Runx2:}

HHHHHHHHHHHHHHHHHHHHH

$\mathrm{HH}$ HHHHHHHH

MLHSPHKQPQNHKCGANFLQEDCKKALAFKWLI SAGHYQP PRPTES / FKAASS I YN

EEEEE EEE HHHHH EEE EEE

RGHKFYLEKKGGTMASNSLFSAVTPCQQSFFW/GCSPRRGTRWDCGYRHGRE

B

testicular Runx2： MLHSPHKQPQNHKCGANFLQEDCKKALAFKWLISAG--HYQPPRPTES Q7TPL8: AFHHKSLLPQYQSARADEQQSDCKELMKIYFYVSSPTQHHGPPPPEKP

C Q7TPL8:

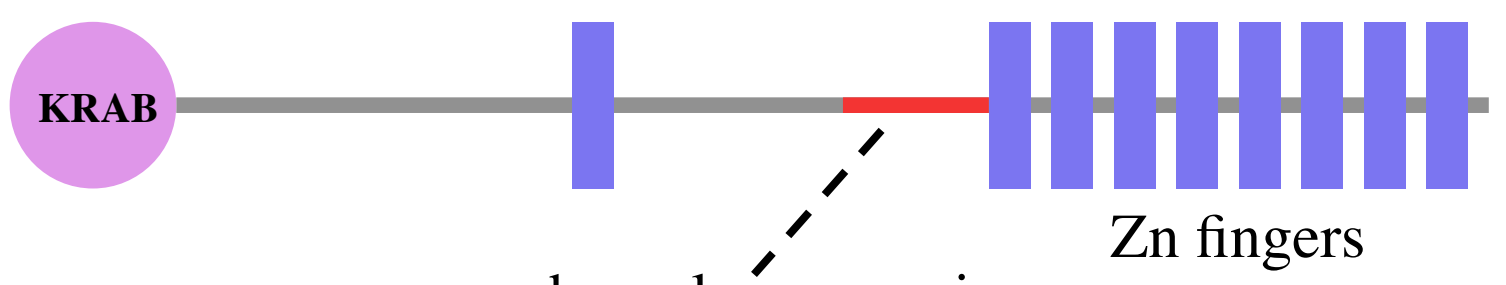

a homologous region 


\section{Figure 4 (on next page)}

Immunoblot detection of Runx2 protein in testis and its localization in nuclei of spermatogenic cells dependent on seminiferous-stage

Fig. 4 - (A) Immunoblot detection of Runx2 is shown. Lanes 1 and 2 contain protein from testes, whereas lanes 3 and 4 contain protein from GST-Runx2-transfected bacteria. The membrane was probed with anti-Runx2 serum, which was preabsorbed with the antigen peptide (lanes 2 and 4) or was not (lanes 1 and 3). The arrow and arrowhead indicate testicular Runx2 and the GST-Runx2 fusion protein, respectively. (B) Immunohistochemical staining of Runx2 protein in testis is shown. Testes from adult C57BL/6] mice were stained with the affinity-purified anti-Runx 2 antibody and counterstained with methyl green. In $b$, the antibody was preabsorbed with the antigen peptide. The yellow scale bars correspond to 251 $\mu \mathrm{m}$. Arrow in panel c: this staining was not disappeared when the antibody was preabsorbed with the antigen peptide. Arrow in panel $d, h$ and i: nuclei of spermatogonia. (C) Enlarged view of positive staining in nuclei is shown. a: pachytene spermatocytes, b: round spermatids. 
A

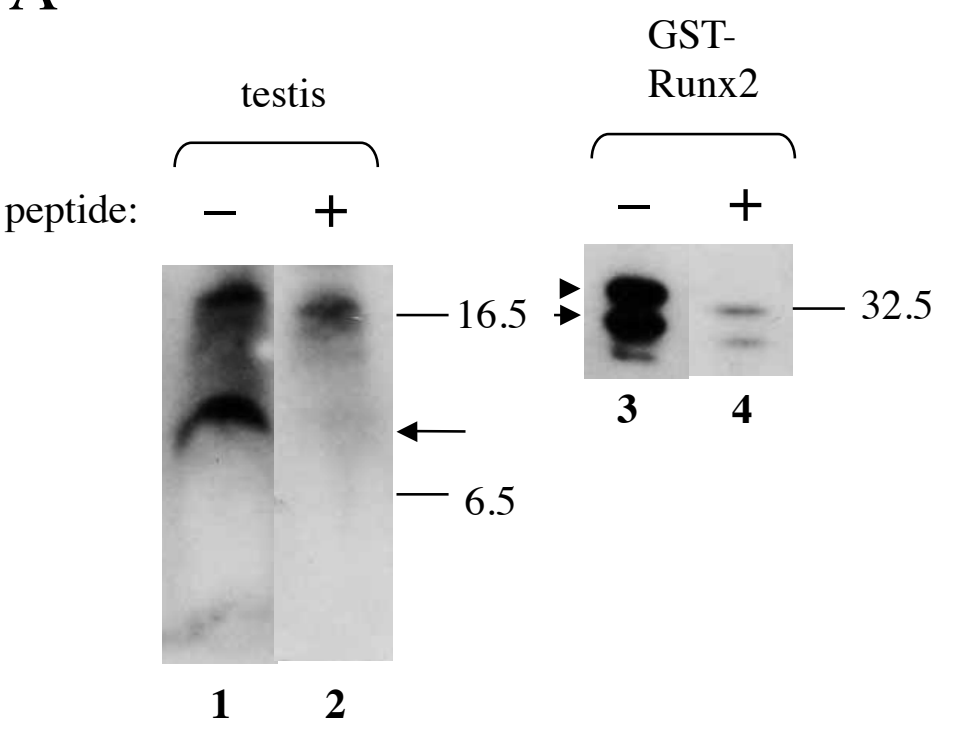

C

(a)

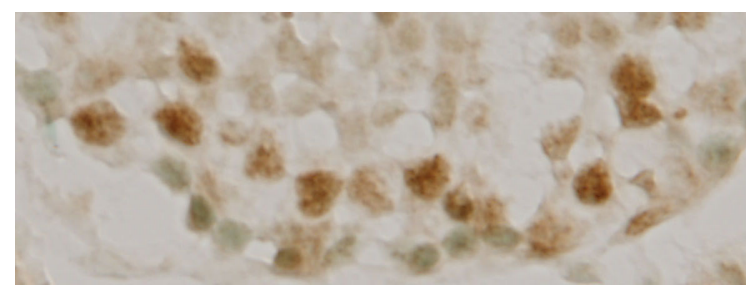

(b)

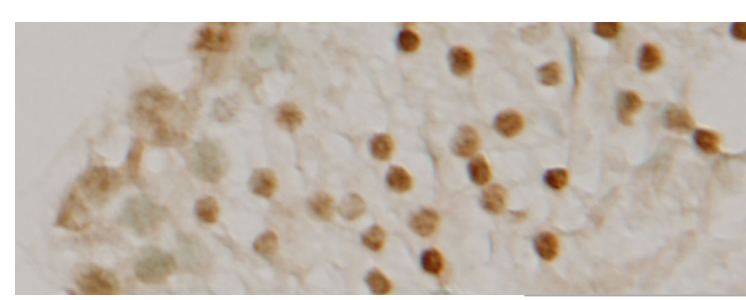

B
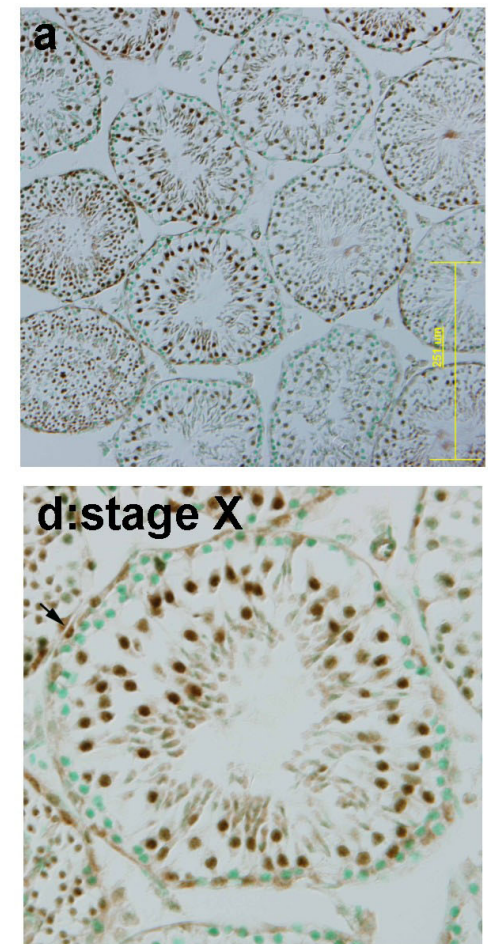

g: stage 1

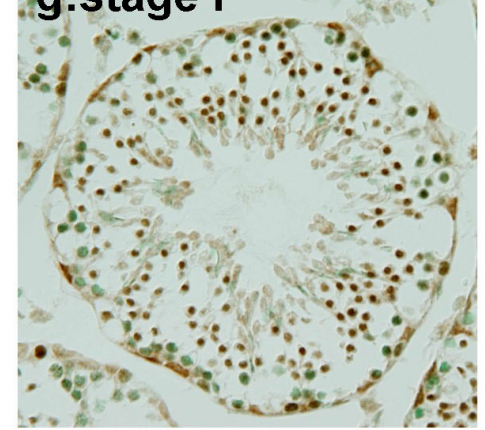

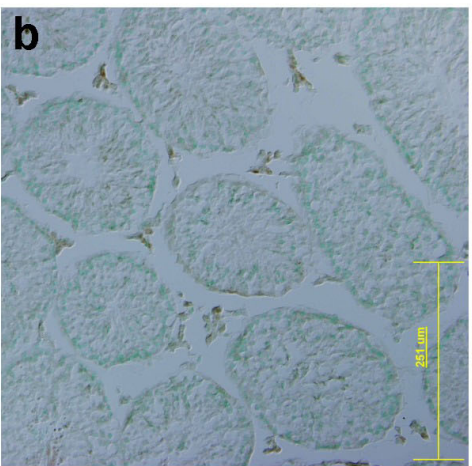
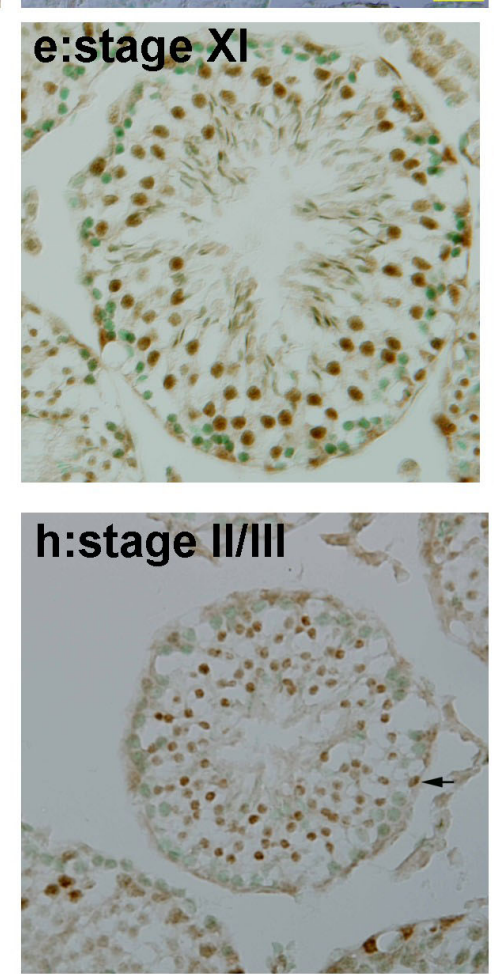
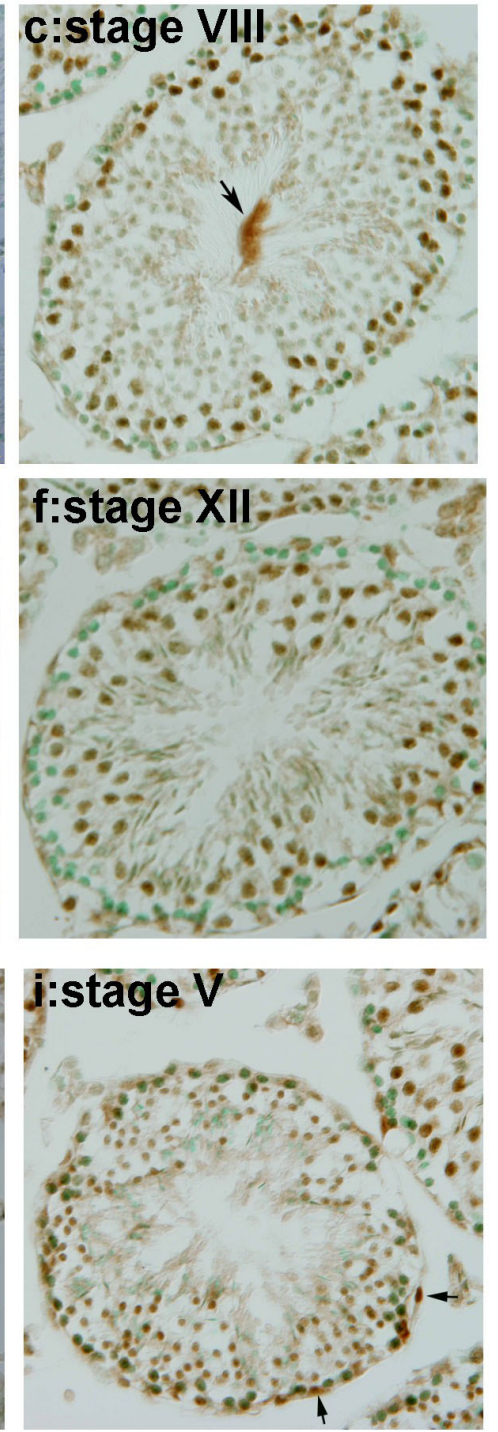